\title{
PARACENTRAL CAPSULE FORCEPS AND A GRID VECTIS
}

BY

\section{J. HORTON YOUNG}

CHELMSFORD

I HAVE detailed below, information of two new instruments, made for me by Messrs. John Weiss and Sons, Ltd., of 287, Oxford Street, W. They are :

(1) Paracentral Capsule Forceps.

(2) A Grid Vectis.

The Paracentral Capsule Forceps.-This instrument represents certain advances on existing instruments and has been designed with due consideration to the anatomical and mechanical factors acting in the operation for extra-capsular extraction of cataract.

The anatomical considerations which induced me to design the instrument were based on the fact that:

(1) The anterior surface of the lens is convex.

(2) The anterior lens capsule is thinnest at the central zone, and thickest in the paracentral zone.

The mechanical factors and disabilities of the other types of capsule forceps were that the straight-toothed blades grasped the lens capsule about the anterior pole. Moreover, the teeth of these forceps were such that they tended to lacerate the anterior capsule into ribbons and leave a great proportion of it behind to form a secondary cataract, thus defeating the purpose and design of the instrument.

The paracentral capsule forceps has thus been designed to eliminate these factors and to meet the anatomical relationships existing in the human eye.

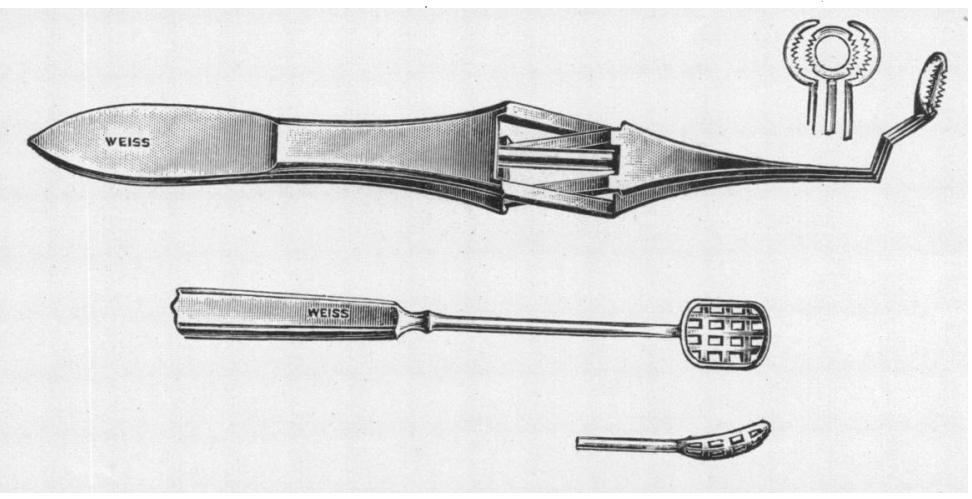


This capsule forceps is based on the cross-action principle for rapidity and ease in operative manipulation. It consists of a body, neck and head.

The body of the instrument forms a tripod the essential elements of which consist of two lateral arms, united in cross-action about a fixed vertical central pin, each of the lateral arms and the central pin ultimately forming part of the head-piece of the forceps.

The cross-action section of the body is placed at its centre so that a double cross-action results.

Normally, on introduction of the head of the instrument into the anterior chamber, the blades of the head are in complete apposition, pressure on the body above the centre-piece separates the blades, while release of pressure enables the spring action of the arms to close the blades and grip the lens capsule. If the fingers are then lowered below the centre-piece and pressure applied, the already held capsule is gripped more firmly. This last action also improves the operative efficiency and ease with which the intra-ocular manipulation can be carried out.

The neck possesses an identically tripled angulation in each of its three elements. The angle near the head is fairly stcep to prevent the free edge of the iris being caught on closure of the blades. This point is of importance in the operative technique, described below. The second angle carries the blades over the iris and through the corneal wound. The final angulation is steep and gets the body of the instrument clear of the globe.

The head of the paracentral capsule forceps is unique and presents the following features. It consists of a central circular ring attached to the fixed central pin, so designed that it engages in the paracentral zone of the lens capsule. Its under-surface is bevelled to fit the curved lens surface while it is armed with (nonlacerating) gripping teeth, turned circumferentially over the greater portion of its extent.

These teeth interdigitate with similar teeth on the mobile lateral jaws, which have curved blades, to approximate the circumference of the first central pin.

The mobile jaws are equipped with gripping teeth, which point axially.

The head of the instrument then gets a double grip of the capsule in the paracentral zone on each side of the central lens axis.

Measured in terms of "linear attachment" the instrument is two and a half times as efficient as other types of capsule forceps. The superior aspect of the head is so excavated that the engaged capsule has room to seat itself without causing an undue separation of the blades.

Operative Technique.-The usual Graefe incision is made and the anterior capsulectomy attempted before iridectomy is done. 
The head of the instrument is inserted through the corneal wound with the smooth upper surface against the under-side of the sectioned cornea.

For the right eye the capsule forceps is held in the left hand, and in the right hand for the left eye. This prevents cramping and allows one to guide the forceps towards the centre of the lens.

As the introduction of the blades takes place, the smooth surface is kept against the cornea and free of the iris.

When the steep angle of the neck of the instrument reaches the circumference of the sectioned cornea, the cornea is made to slide gently up the neck by tilting the body of the instrument back slightly. This will bring the central axis of the fixed centre pin over the central axis of the lens.

The blades are then separated and made to engage the capsule, and the capsule separated by lateral tearing movement.

The removal of the forceps with the engaged capsule is the reverse of the above manipulation of introduction.

The Grid Vectis.-This instrument consists of the usual vectis loop into which has been inserted a fine mesh.

The mesh (or grid) has been designed to allow aqueous and degenerate vitreous to sieve through, but not thickened lens matter.

The grid prevents the escape from the vectis of dislocated lenses situated in awkward positions within the globe. It prevents "doubling" through the loop of a shrunken lens, and enables the operator to secure the nucleus of the hypermature cataract.

I have known the vectis cut the lens in two and the problem of removing the portions is almost insuperable. The grid will prevent this.

Such conditions as friable foreign bodies in the anterior chamber can now be attempted with some hope of success. Such foreign bodies include sandstone, coal and glass.

The vectis head is saucer shaped, attached to a malleable silver stem, so that the head can be adjusted to any angle to suit the needs of the surgeon.

\section{THE COUNCIL OF BRITISH OPHTHALMOLOGISTS}

\section{Annual Report 1938-39}

The Council presents its report for the year 1938-39. At its meeting in July the following were elected Officers:-President: Sir John Parsons; Vice-Presidents: Mr. R. A. Greeves and Mr. W. H. McMullen; Hon. Treasurer: Mr. Humphrey Neame; Hon. Secretary: Mr. M. L. Hepburn. 